\title{
The value of middle cerebral artery to umbilical artery ratio by Doppler velocimetry in low risk postdate pregnancies
}

\section{Abstract}

Background and objective: Placental insufficiency is the primary cause of intrauterine growth restriction in normally formed fetuses and can be identified using middle cerebral artery to umbilical artery ratio Doppler velocimetry, and provide an estimate of downstream placental vascular resistance and placental blood flow. There is a strong association between reduced end-diastolic umbilical artery blood flow velocity and increased vascular resistance in umbilical placental microcirculation. Doppler ultrasound can assess the uteroplacental blood flow just before labor. This study aimed to investigate the use of the fetal cerebroumbilical ratio to predict the intrapartum fetal compromise in appropriately grown fetuses.

Methods: A comparative cross-sectional study set at Sulaimania Maternity Teaching Hospital, Sulaimania, Iraq, from January to June 2015. The study recruited 121 cases, fetal biometry and Doppler indices were measured before established labor. The intrapartum and neonatal outcome details recorded.

Results: Infants delivered by cesarean section for fetal compromise had significantly lower cerebroumbilical ratio than those born by spontaneous normal (none assisted) vaginal delivery and by cesarean section for other intrapartum causes. Infants with cerebroumbilical ratio $<10^{\text {th }}$ percentile were more likely to be delivered by cesarean section for fetal compromised than those with a cerebroumbilical ratio $\geq 10^{\text {th }}$ percentile. A cerebroumbilical ratio $>90^{\text {th }}$ percentile appears protective against cesarean section for fetal compromise. Amniotic fluid index of $<5$ was associated with an increased cesarean section for fetal indication.

Conclusion The cerebroumbilical ratio can identify fetuses at high risk of intrapartum fetal compromise. As a confounding variable, the amniotic fluid index was a useful tool for surveillance in prolonged pregnancy.

Keywords: Cerebralumbilical ratio; Amniotic fluid index; Doppler; Labour.

\section{Introduction}

Postdate pregnancy is a common problem. Its incidence has been reported to be between $4-14 \%$ with an average of $10.5 \%{ }^{1}$ A safe limit for the continuation of pregnancy beyond the expected date of delivery cannot be established, and there is also controversy on whether the risk of fetal hypoxia can be accurately predicted in these pregnancies. Rayburn and Chang suggested that the risk of postmaturity starts at 40 weeks. $^{2}$ Postdate pregnancies have been associated with increased perinatal morbidity and mortality up to 42 weeks and these risks increase after 42 weeks (post-term pregnancy). ${ }^{3}$ Increased incidence of induction of labor, instrumental delivery, cesarean section, shoulder dystocia, lower Apgar score, congenital malformation, meconium aspiration, and fetal asphyxia have been associated with these pregnancies., These problems can be decreased by routine antepartum fetal surveillance prior to the onset of spontaneous labor. ${ }^{5,6}$ The current methods of fetal surveillance like

* Department of Obstetric/Gynecology, College of Medicine, University of Sulaimani, Sulaimani, Iraq. ** Department of Health System, Kirkuk, Iraq. 
nonstress test (NST), amniotic fluid index (AFI), biophysical score (BPS), umbilical artery (UA) S/D ratio and middle cerebral artery (MCA) plasticity index $(\mathrm{PI})$ cannot accurately predict fetus at risk of the adverse perinatal outcome. ${ }^{3,6}$ Various studies have investigated MCA, and UA (cerebroumbilical (CU) ratio) in post-term pregnancies with high risk complicating factors like chronic hypertension, pregnancy induced hypertension $(\mathrm{PIH})$ and diabetes, and found it to accurately predict fetal compromise. ${ }^{7,8}$ These conditions, however, are known to affect the vascular bed and placental circulation, the blood flow to the fetus. Very few studies have been done on the value of middle cerebral artery to umbilical artery ratio (CU ratio) in determining the perinatal outcome in low risk postdate pregnancies. ${ }^{8}$ The incidence of postdate pregnancy is higher in first pregnancies and in women who have had a previous postdate pregnancy. Genetic factors may also play a role. One study showed an increased risk of postdate pregnancy in women who were, themselves, born postdate. ${ }^{9}$ An ultrasound examination performed in the first half of pregnancy is the most reliable method of calculating the date the baby is due, especially in women with long or irregular menstrual cycles. ${ }^{10}$ The American College of Obstetricians and Gynecologists has stated that it is only necessary to start antenatal fetal monitoring after 42 weeks (294 days) of gestation, although many obstetric care providers will start fetal testing at 41 weeks. Many experts recommend twice weekly testing, including measurement of amniotic fluid volume. Testing may include observing the fetus' heart rate using CTG, or observing the baby's activity by biophysical profile. ${ }^{11}$ Several studies have suggested that oligohydramnios detected by ultrasound is a useful test for detecting placental insufficiency, ${ }^{12,13}$ Although AFI is of limited value in identifying fetuses at risk of compromise. ${ }^{14} \mathrm{~A}$ good liquor volume is a reassuring sign that the fetus has not been subjected to chronic hypoxia in the antenatal period ${ }^{15}$ and this was reflected in our study.

Doppler velocimetry: The presence of forward flow during diastole is seen in arteries supplying low resistance vascular beds. Diastolic components disappear or reverse as the peripheral impedance increases. ${ }^{16}$ Different indices have been proposed to define the properties of the Doppler spectrum. The most common in the obstetric application being the pulsatility index, resistance index and the Systolic /Diastolic (S/D) ratio. ${ }^{16}$ The closer the measurement site is to the placenta, the less is the wave reflection and the greater the end -diastolic flow. Consequently, the Doppler waveform that represents arterial flow velocity demonstrates progressively declining pulsatility and the indices of pulsatility from the fetal to the placental end of the cord. ${ }^{17}$ Meta-analysis of the previously published randomized controlled studies clearly indicates that UA velocimetry assessment decreases the perinatal mortality from intrauterine growth retardation (IUGR) without any increase in the rate of unnecessary obstetric interventions in high -risk pregnancies. Knowledge of Doppler flow velocimetry of the fetal Middle cerebral artery (MCA) may assist in perinatal diagnosis and management of complicated pregnancies and high risk pregnancy. A low index of pulsatility in the middle cerebral artery associated with fetal compromise has been described. ${ }^{18}$ Because the fetal Middle cerebral artery to umbilical artery (MCA/UA) ratio incorporates data not only on placental status but also on the fetal response, it is potentially more advantageous in predicting the perinatal outcome. Doppler data combining both umbilical and cerebral velocimetry provide additional information on fetal consequences of the placental abnormality. ${ }^{19}$ The current data, however, question the benefits of using CU Arterial Doppler velocimetry ratio as a routine screening test for fetal hypoxia or acidosis 
in low-risk pregnancies. ${ }^{20}$ This study was designed to study the Doppler waveforms in UA and MCA, and CU ratio in uncomplicated postdate pregnancies, and to correlate these findings with the perinatal outcome. It also aimed to determine the cutoff value of $\mathrm{CU}$ ratio for predicting adverse perinatal outcome in these pregnancies.

\section{Methods}

One hundred and twenty one women with postdate pregnancy (40 weeks pulse) were recruited at Maternity teaching hospital in Sulaimania, Iraq from first January 2015 to first June 2015. All patients recruited before active labor (cervical dilatation $\leq 4 \mathrm{~cm}$ ), had singleton pregnancies and were identified as low risk and uneventful pregnancy. Information about maternal age, ethnicity, parity, gestation at the onset of labor, body mass index (BMI), smoking, preexisting maternal medical disorders, history of previous fetal growth restriction, stillbirth, or neonatal death were documented. The inclusion criteria included singleton cephalic presenting pregnancy, more than 40 weeks and less than 42 weeks, no current medical disease or medical disease complicating pregnancy, either presented in spontaneous labor or been induced for labor, and cervical dilation less than $4 \mathrm{~cm}$. The exclusion criteria included pregnancy $<40$ week, cervical dilatation $>4 \mathrm{~cm}$, malpresentation, membrane rupture, multiple pregnancy, preeclampsia, previously identified fetal growth restriction, known fetal anomaly, and evidence of intrauterine infection. All women had an ultrasound (US) examination performed in a supine position with the head of the bed elevated at about 45 degrees. Fetal biometry and AFI and FHR were recorded. In addition, the resistant index (RI) and pulsatility index (PI) of the umbilical artery (UA) and middle cerebral artery (MCA) were recorded using a manual trace of one proper waveforms. Each parameter was recorded twice and a mean of these values used for data analysis. Umbilical artery Doppler studies were obtained from a mid-segment of the umbilical cord. All US examinations were undertaken by using (SIEMENS - SONOLINE G50) Machine. Labour was then managed according to local protocols and guidelines. Outcome measures for this study were collected including intrapartum data were diagnosis of fetal compromise (based on abnormal FHR during electronic fetal heart monitoring or CTG abnormalities), and presence of meconium stained liquor and mode of delivery, and neonatal outcome were assessed by examining birth weight, Apgar score at the $1^{\text {st }}$ and $5^{\text {th }}$ minutes, admission to neonatal care unit. Data analysis was performed using the statistical package for the social sciences (version 20), and Microsoft Word and Excel have been used to generate graphs and tables.

\section{Results}

A comparative cross-sectional study of 121 intrapartum women was done after considering the inclusion and exclusion criteria. RI and PI index of the umbilical, middle cerebral arteries and the ratio of cerebro-umbilical arteries were obtained from all the 121cases. Data were analyzed. The mean \pm SD of maternal age, and BMI of the studied group seen in Table 1.

Table 1: Mean age, birth weight, and BMI of study population.

\begin{tabular}{lcc}
\hline Parameters & Mean \pm SD & Range \\
\hline Age, year & $27.7 \pm 5.7$ & $18-41$ \\
BMl, kg/ht & $22.8 \pm 2.3$ & $18-29$ \\
\hline
\end{tabular}


Means \pm SD of specific parameters RI respectively. The highest $1^{\text {st }}$ minute ratio, $\mathrm{PI}$ ratio, $\mathrm{AFI}$, and birth weight was seen in Table 2 . The results show $56.2 \%$ of the woman had a vaginal delivery, and $43.8 \%$ of woman had an emergency cesarean section. Most of the infants in this study were female in $60.3 \%$. Admission to $\mathrm{NCU}$ and meconium aspiration syndrome occurred in $53.7 \%$ and $2.5 \%$ of infants, APGAR score was 3 and 5 in $17.4 \%$ and $18.2 \%$, respectively, and the $5^{\text {th }}$ minute Apgar score was 9 and 10 in $20.7 \%$ and $38.0 \%$, respectively. Significant differences were found in RI ratio in women who had a vaginal delivery, meconium stained liquor, emergency CS, and admission to NCU, $P<0.01$ (Table 3)

Table 2: Means \pm SD of specific parameters in studied group.

\begin{tabular}{lcc}
\hline Parameters & Mean \pm SD & Range \\
\hline RI ratio & $1.2 \pm 0.2$ & $0.4-1.9$ \\
PI ratio & $1.4 \pm 0.4$ & $0.9-2.7$ \\
AFI & $9.5 \pm 3.7$ & $1.0-23.0$ \\
Birth weight, mg & $3343 \pm 459$ & $2300-5000$ \\
\hline
\end{tabular}

Table 3: RI ratios in the parameters of studied group.

\begin{tabular}{lcc}
\hline Parameters & $\begin{array}{c}\text { RI ratio } \\
\text { Mean } \pm \text { SD }\end{array}$ & $P$ value \\
\hline Vaginal delivery & $1.23 \pm 0.19$ & \\
Yes & $1.06 \pm 0.22$ & 0.001 \\
No & $1.07 \pm 0.22$ & \\
Meconium stained liqour & $1.23 \pm 0.20$ & 0.001 \\
Yes & $1.03 \pm 0.19$ & \\
No & $1.24 \pm 0.21$ & 0.001 \\
Emergency cs of fetal indication & & 0.001 \\
Yes & $1.09 \pm 0.23$ & \\
No & $1.24 \pm 0.21$ & 0.850 \\
Need for admission to NCU & & \\
Yes & $1.14 \pm 0.11$ & \\
No & $1.16 \pm 0.23$ & \\
Meconium aspiration syndrome & & \\
Yes & & \\
No & & \\
\hline
\end{tabular}


A significant positive correlation was found in this study between the RI ratio and $1^{\text {st }}$ minute Apgar score and $5^{\text {th }}$ minute Apgar score, (Table 4). Highly significant differences were found in PI ratio in women who had a vaginal delivery, meconium stained liquor, emergency CS for fetal indication, and admission to NCU, $P<0.01$ (Table 5). A significant positive correlation was found in this study between the PI ratio and $1^{\text {st }}$ minute Apgar score and $5^{\text {th }}$ minute Apgar score (Table 6).

Table 4: Correlation coefficient between RI ratio and other parameters in studied group.

\begin{tabular}{lcc}
\hline Parameters & Correlation coefficient $(\boldsymbol{r})$ & $\boldsymbol{P}$ value \\
\hline RI ratio: Birth weight & 0.048 & 0.600 \\
RI ratio: $1^{\text {st }}$ minute Apgar score & 0.372 & 0.001 \\
RI ratio: $5^{\text {th }}$ minute Apgar score & 0.335 & 0.001 \\
\hline
\end{tabular}

Table 5: PI ratios in the parameters of studied group.

\begin{tabular}{lcc}
\hline Parameters & PI ratio Mean \pm SD & $P$ value \\
\hline Vaginal delivery & $1.52 \pm 0.36$ & \\
Yes & $1.22 \pm 0.39$ & 0.001 \\
No & & \\
Meconium stained liquor & $1.24 \pm 0.39$ & 0.001 \\
Yes & $1.51 \pm 0.37$ & \\
No & & \\
Emergency cs for fetal indication & $1.16 \pm 0.33$ & 0.001 \\
Yes & $1.54 \pm 0.37$ & \\
No & & \\
Need for admission to NCU & $1.26 \pm 0.39$ & \\
Yes & $1.53 \pm 0.37$ & \\
No & & \\
Meconium Aspiration Syndrome & $1.40 \pm 0.09$ & \\
Yes & $1.39 \pm 0.40$ & \\
No & & \\
Miscarriage & $1.38 \pm 0.39$ & 0.900 \\
Yes & $1.39 \pm 0.46$ & \\
No & & \\
\hline
\end{tabular}

Table 6: Correlation coefficient between PI ratio and other parameters in studied group.

\begin{tabular}{lcc}
\hline Parameters & Correlation coefficient $(\mathbf{r})$ & $\boldsymbol{P}$ value \\
\hline $\begin{array}{l}\text { PI ratio } \\
\text { Birth weight }\end{array}$ & 0.054 & 0.553 \\
$\begin{array}{l}\mathrm{PI} \text { ratio } \\
1^{\text {st }} \text { minute Apgar score }\end{array}$ & 0.379 & 0.001 \\
$\mathbf{P I}_{\text {ratio }}^{\text {th }}$ minute Apgar score & 0.780 & 0.001 \\
\hline
\end{tabular}




\section{Discussion}

This comparative cross-sectional study was conducted to evaluate cerebral and umbilical arterial blood flow velocity in low risk postdate pregnancy. The study results demonstrated that the assessment of the fetal $\mathrm{C} / \mathrm{U}$ ratio, in postdate low risk pregnancies before active labor, may predict the diagnosis of intrapartum fetal compromise (based on abnormal CTG) and the need for emergency delivery. Currently, multi vessel Doppler assessment of fetal well-being is considered valuable only in cases of fetal growth restriction. Our data suggest that assessment of fetal $\mathrm{C} / \mathrm{U}$ ratio can also be of value in the apparently normal grown fetuses beyond term. Where the risk of intrapartum fetal compromise is highest in infants with the lowest $\mathrm{C} / \mathrm{U}$ ratios, and a high $\mathrm{C} / \mathrm{U}$ ratio appears to suggest, better fetal tolerance to the stresses of labor, with a reduced incidence of abnormal fetal heart rate patterns necessitating emergency delivery. Only $15(17 \%)$ infant in this study with a $\mathrm{C} / \mathrm{U}$ ratio $>90^{\text {th }}$ percentile required delivery by emergency CS for fetal heart rate abnormalities. Numerous studies with varying results have been published and difficult to compare. Variation in sample sizes and techniques, as well as different criteria like fetal blood sampling and cord $\mathrm{PHs}$, used to define the adverse perinatal outcome. A study that performed in 1988 by Ferrazzi demonstrates that raised UAPI is associated with increased placental resistance and has previously been suggested as a surrogate marker of placental function. ${ }^{21}$ Another study by Farrell that performed in 1999 found that UA PI is known to be elevated in cases of fetal growth restriction. However, its use in early labor does not appear to be a good predictor of adverse perinatal outcome. ${ }^{22}$ In 1999 a study that performed by Bahado-Singh on 203 fetuses at risk for intrauterine growth restriction, there was a statistically significant increase in perinatal morbidity and mortality in cases with an abnormal cerebroumbilical ratio. ${ }^{23}$ In addition, some studies were performed in high risk and some in low risk population. Our study agreed with a similar study conducted in London Queen Charlotte's and Chelsea hospital by Tomas Prior in 2013 the study population was a group of women aged 18-47years, values and results close to this study have been observed, in the Tomas study 2013, other parameters were observed also; as body mass index, gestational age by ultrasound, parity, mode of delivery and CTG result and type of the liquor. The relation between them and mode of delivery was found, and results are close to what has been computed in this study. The results of umbilical artery $\mathrm{PI}$ and Middle cerebral artery PI was interesting as in the Tomas study the relationship between them and the intrapartum fetal outcome was significant and this is in agreement with our results. There are other studies performed in India one of them performed by Bindu in 2012 on the cerebral and umbilical blood flow velocity in normal and growth retarded babies. In the mentioned study two groups of mothers have been followed, one group had normal pregnancies without risk factors, this acted as a control group, and the other was on IUGR. The middle cerebral artery $\mathrm{PI}$ were not significant between the two groups $P=0.3$, but for umbilical artery $\mathrm{PI}$ were significant $P=0.03$. Similarly, $C U$ was significant between two groups $P=0.01$. This shows the importance of $\mathrm{C} / \mathrm{U}$ as a predictor of fetal compromise. ${ }^{24}$ In this study, we have observed that there was a strong relation of meconium stain liquor with the $\mathrm{C} / \mathrm{U}$ ratio. This finding agrees with the finding of the study done by Tomas Prior that studied in $2013,{ }^{25}$ which revealed a higher rate of meconium stained liquor in infants with the lowest $\mathrm{C} / \mathrm{U}$ ratio $(0.76)$. While $\mathrm{Lam}^{25}$ in 2005, who evaluated the use of AFI, MCA $\mathrm{PI}, \mathrm{UA} \mathrm{PI}$, and $\mathrm{C} / \mathrm{U}$ ratio in surveillance of postdates pregnancy, also found that MCA $\mathrm{PI}$ was the only parameter that had a statistically significant correlation with grade three or thick meconium stained 
liquor. ${ }^{25}$ Another study that performed by Mishra in $2013^{26}$ showed that Doppler flow velocimetry of the fetal MCA may assist in perinatal diagnosis and management of complicated pregnancies. A low index of pulsatility in the middle cerebral artery associated with fetal compromise. Because the MCA/UA ratio incorporates data not only on placental status but also on the fetal response; it is potentially more advantageous in predicting the perinatal outcome. Doppler data combining both umbilical and cerebral velocimetry provide additional information on fetal consequences of the placental abnormality. ${ }^{26}$ In another study that performed in 2013 by Rajesh M. found that the cerebral/umbilical pulsatility ratio $(\mathrm{C} / \mathrm{U}$ ratio) has been recognized as the more sensitive and specific indicator of likelihood of IUGR and adverse perinatal. ${ }^{27}$ The result of this study suggests that the fetal $\mathrm{C} / \mathrm{U}$ ratio, measured before established labor, can predict the diagnosis of intrapartum fetal compromise, and is a better predictor of adverse perinatal outcome than an abnormal MCA PI or UA PI alone. The mean values of $\mathrm{CU} \mathrm{RI}$ and $\mathrm{CU} \mathrm{PI}$ ratio were 1.2 and 1.4 respectively. Placio et al. ${ }^{28}$ observed mean $\mathrm{CU}$ ratio to be 1.36 at 41 weeks and 1.27 at 42 weeks. In the present study, a cutoff value of CU ratio of 1 was obtained and used for correlating perinatal outcome in these postdate pregnancies. The CU ratio of 1 for predicting adverse perinatal outcome in the present study was similar to the value established by Devine et al. ${ }^{29}$ The authors observed that $\mathrm{CU}$ ratio in their study had high specificity and positive predictive value for determining the adverse perinatal outcome. This difference appears to be because they included high risk postdate pregnancies with complicating factors like diabetes, chronic hypertension, and $\mathrm{PIH}$ unlike in the present study where only uncomplicated postdate pregnancies were enrolled.

\section{Conclusion}

Cerebro umbilical ratio (MCA/UA $\mathrm{PI})$ is a good predictor for adverse perinatal outcome during intrapartum care and can be used as a tool to guide the mode of delivery in the low risk pregnancy. Can be considered as a useful technique for fetal surveillance in prolonged pregnancy.

\section{Competing interests}

The authors declare that they have no competing interests.

\section{References}

1. Bakketeig L, Bergsjo P. Post-term pregnancy magnitude of the problem. In: Chalmers I, Elkin M, Kierse MJNC (Eds). Effectivecare in pregnancy and childbirth. London: Oxford University Press; 1991. P. 765-75.

2. Rayburn WF, Chang FE. Management of the uncomplicated postdate pregnancy. J Reprod Med 1981; 26:93-5.

3. Hollis B. Prolonged pregnancy. Curr Opin Obstet Gynecol 2002; 14:203-7.

4. Clausson $B$, Cnattingius $S$, Axelsson $\mathrm{O}$. Outcomes of post-term births: the role of fetal growth restriction and malformations. Obstet Gynecol 1999; 94:758-62.

5. Arias F. Predictibility of complications associated with prolongation of pregnancy. Obstet Gynecol 1987; 70:101-6.

6. Shime J, Gare DJ, Andrews J, Prolonged pregnancy: surveillance of the fetus and the neonate and the course of labor and delivery. Am J Obstet Gynecol 1984; 148:547-52.

7. Arias F. Accuracy of middle-cerebral to umbilical artery resistance index ratio in the prediction of neonatal outcome in patients at high risk for fetal and neonatal complications. Am J Obstet Gynecol 1994; 171:1541-5.

8. Gramellini D, Folli MC, Raboni S, Cerebral umbilical Doppler ratio as a predictor of adverse perinatal outcome. Obstet Gynecol 1992; 79:416 -20 .

9. Mogren I, Stenlund H, Hogberg U. Recurrence of prolonged pregnancy. Int J Epidemiol 1999; 28:253-257.

10. Antenatal Care, Routine Care for the Healthy Pregnant WomanNICE Clinical Guidelines, No. 62 National Collaborating Centre for Women's and Children's Health (UK). London: RCOG Press; 2008.

11. Roos N, Sahlin L, Ekman-Ordeberg G. Maternal risk factors for postterm pregnancy and cesarean delivery following labor induction. Acta Obstet Gynecol Scand. 2010; 89(8):100310.

12. O'Reilly-Green CP. Divon MY. Predictive value of amniotic fluid index for oligohydramnios in patients with prolonged pregnancies. J Matern Fetal Med 1996; 5:218-26. 
13. Rainford M. Adair R. Scialli AR. Ghidini A. Spong CY. Amniotic fluid index in the uncomplicated term pregnancy: prediction of outcome. J Reprod Med 2001; 46:589-92.

14. Prior T, Mullins E, Bennett $\mathrm{P}$, Kumar S. Prediction of intrapartum fetal compromise using cerebroumbilica ratio: aprospective observational study. Am J Obstet Gynecol 2013; 208(2):124e16.

15. Luesley DM, Baker PN. Obstetrics and Gynecology: An evidence-based text for MRCOG. $2^{\text {nd }}$ ed. London: Hodder Arnold; 2010. P. 381-8.

16. Reddy D. Value of colour Doppler in evaluation of intrauterine growth restriction with clinical correlation. MD thesis in Radiodaignosis. Bangalore, India; Rajiv Gahndi University of Health Sciences: 2008.

17. Maulik D, Yarlagadda P, Downing G. Doppler velocimetry in obstetrics. Obstet Gynecol 1989; 32:628-44.

18. Madazli R, Uludağ S, Ocak V. Doppler assessment of umbilical artery, thoracic aorta and middle cerebral artery in the management of pregnancies with growth restriction. Acta Obstet Gynecol Scand 2001; 80(8):702-7.

19. Bahado-Singh RO, Kovanci $E$, Jeffres $A$, Oz U, Deren O, Copel J, Mari G. The Doppler cerebroplacental ratio and perinatal outcome in intrauterine growth restriction. Am $\mathrm{J}$ Obstet Gynecol 1999; 180(3 Pt 1):750-6.

20. Callen PW. Callen ultrasonography in obstetrics and gynecology. 4th ed., Philadelphia: Saunders; 2000. P. 686-92.

21. Ferrazzi E, Pardi G, Bauscaglia M, Marconi AM, Gementi B, Bellotti M, et al. The correlation of biochemical monitoring versus umbilical flow velocity measurements of the humanfetus. Am J Obstet Gynecol 1988; 159:1081-7.

22. Farrell T, Chien PF, Gordon A. Intrapartum umbilical artery Doppler velocimetry as a predictor of adverse perinatal outcome: a systematic review. Br J Obstet Gynaecol 1999; 106:783-92.

23. Bahado- Singh RO, Kovanci E, Jeffres A, Oz U, DerenO, Copel J, Mari G. The Doppler cerebroumbilical ratio and perinatal outcome in intrauterine growth restriction. Am J Obstet Gynecol 1999;180(3,1):750-6.

24. Bindu T. Cerebral and umbilical arterial blood flow velocity in normal and growth retarded pregnancy. MSc thesis in Obstetrics and Gynecology. Bangalore, India: Rajiv Gandhi University of Health Sciences; 2012.

25. Lam H, Leung WC, Lee CP, Lao TT. The use of fetal Doppler cerebroplacental blood flow and amniotic fluid volume measurement in the surveillance of postdated pregnancies. Acta Obstet Gynecol Scand 2005; 84:844-8.

26. Mishra D, Sakhi D, Saraf D, Gupta D, Soni D, Dhruwr D. Role of Obstetric Doppler in Prediction of Adverse Perinatal Outcome in Intrauterine Growth Retardation and Pregnancy Induced Hypertension. SJAMS 2013; 1(6):1016-20.

27. Rajesh M, Agamya S.Role of Colour Doppler Indices in the Diagnosis of Intrauterine Growth Retardation in High-Risk Pregnancies. The Journal of Obstetrics and Gynecology of India 2013; 63(1):37-44.

28. Palacio $M$, Figueras $F$, Zamora $L$, Jiménez JM, Puerto B, Coll O, et al. Reference ranges for umbilical and middle ceretral artery pulsatility index and cerebroplacental ratio in prolonged pregnancies. Ultrasound Obstet Gynecol 2004: 24:647-53.

29. Devine PA, Bracero LA, Lysikiewicz A, Evans R, Womack S, Byrne DW. . Middle cerebral to umbilical artery Doppler ratio in post-date pregnancies. Obstet Gynecol 1994; 84:856-60. 